\section{Roles of PINK1, mTORC2, and mitochondria in preserving brain tumor-forming stem cells in a noncanonical Notch signaling pathway}

\author{
Kyu-Sun Lee, ${ }^{1,2,8}$ Zhihao $\mathrm{Wu}^{1,8}$ Yan Song, ${ }^{1,3,4}$ \\ Siddhartha S. Mitra, ${ }^{5,6}$ Abdullah H. Feroze, 5,6,7 \\ Samuel H. Cheshier, ${ }^{5,6,7}$ and Bingwei $\mathrm{Lu}^{1,9}$ \\ ${ }^{1}$ Department of Pathology, Stanford University School \\ of Medicine, Stanford, California 94305, USA; \\ ${ }^{2}$ BioNanotechnology Research Center, Korea Research Institute \\ of Bioscience and Biotechnology, Daejeon 305-806, Korea; \\ ${ }^{3}$ School of Life Sciences, ${ }^{4}$ Peking-Tsinghua Center for Life \\ Sciences, Peking University, Beijing 100871, China; ${ }^{5}$ Institute of \\ Stem Cell Biology and Regenerative Medicine, ${ }^{6}$ Stanford Ludwig \\ Center for Cancer Stem Cell Research and Medicine, \\ ${ }^{7}$ Department of Neurosurgery, Stanford University School \\ of Medicine, Stanford, California 94305, USA
}

The self-renewal versus differentiation choice of Drosophila and mammalian neural stem cells (NSCs) requires Notch $(\mathbf{N})$ signaling. How $\mathbf{N}$ regulates NSC behavior is not well understood. Here we show that canonical $\mathbf{N}$ signaling cooperates with a noncanonical $\mathbf{N}$ signaling pathway to mediate $\mathrm{N}$-directed NSC regulation. In the noncanonical pathway, $\mathbf{N}$ interacts with PTEN-induced kinase 1 (PINK1) to influence mitochondrial function, activating mechanistic target of rapamycin complex 2 (mTORC2)/AKT signaling. Importantly, attenuating noncanonical $\mathrm{N}$ signaling preferentially impaired the maintenance of Drosophila and human cancer stem cell-like tumor-forming cells. Our results emphasize the importance of mitochondria to N and NSC biology, with important implications for diseases associated with aberrant N signaling.

Supplemental material is available for this article.

Received June 22, 2013; revised version accepted November 6, 2013.

Maintaining a delicate balance between self-renewal and differentiation is a hallmark of all stem cells (Morrison and Kimble 2006; Doe 2008; Zhong and Chia 2008). Impairments of such balance can lead to lineage depletion or tumorigenesis. The self-renewal versus differentiation decision of mammalian neural stem cells (NSCs) and Drosophila neuroblasts (NBs), an excellent model for NSC biology (Doe 2008; Knoblich 2010; Sousa-Nunes et al. 2010), requires Notch (N) signaling (Wang et al.

[Keywords: Notch; PINK1; mTORC2/AKT signaling; mitochondria; neural stem cells; cancer stem cells]

${ }^{8}$ These authors contributed equally to this work.

${ }^{9}$ Corresponding author

E-mail bingwei@stanford.edu

Article is online at http://www.genesdev.org/cgi/doi/10.1101/gad.225169.113.
2006; Artavanis-Tsakonas and Muskavitch 2010; Andersson et al. 2011). In the type II NB lineages of the Drosophila larval central brain, which contain transit-amplifying intermediate progenitors (IPs) and are similar to mammalian NSCs in lineage hierarchy (Fig. 1A), inhibition of $\mathrm{N}$ signaling leads to NB loss, whereas $\mathrm{N}$ activation causes the dedifferentiation of IPs into ectopic NBs (Bowman et al. 2008; Weng et al. 2010; Song and Lu 2011), reminiscent of the cell of origin of brain tumors in mammals (Dirks 2010; Liu and Zong 2012). The mechanism by which $\mathrm{N}$ signaling maintains NB lineage homeostasis is not well defined. N can signal through Suppressor of Hairless $[\mathrm{Su}(\mathrm{H})]$ to transcriptionally regulate its target gene, Myc, whose regulation of cell growth is critical for the maintenance of NSCs and cancer stem cell (CSC)-like cells in Drosophila. However, overexpression of Myc alone is insufficient to mimic the effect of $\mathrm{N}$ in promoting ectopic NSC formation (Song and Lu 2011), suggesting the involvement of other pathways.

Here we show that a novel noncanonical $\mathrm{N}$ signaling pathway is involved in N-directed NSC regulation. In this pathway, $\mathrm{N}$ interacts with PTEN-induced kinase 1 (PINK1) to influence mitochondrial function, activating mechanistic target of rapamycin complex 2 (mTORC2)/ AKT signaling and enhancing NB growth and proliferation. Importantly, attenuating the noncanonical $\mathrm{N}$ signaling pathway preferentially impaired the maintenance of Drosophila and human brain CSC-like cells. Canonical $\mathrm{N}$ signaling, which promotes nucleolar growth, acted together with the newly identified noncanonical $\mathrm{N}$ signaling pathway to maintain normal NBs. Moreover, coactivation of canonical and noncanonical $\mathrm{N}$ signaling was sufficient to induce the dedifferentiation of IPs into ectopic NBs, recapitulating the effect of $\mathrm{N}$ activation. Our results identify a noncanonical $\mathrm{N}$ signaling pathway preferentially required by brain CSC-like cells, emphasize the underappreciated importance of mitochondria in $\mathrm{N}$ and stem cell biology, and have important implications for cancer and other diseases caused by aberrant $\mathrm{N}$ signaling.

\section{Results and Discussion}

To test whether canonical $\mathrm{N}$ signaling is sufficient to account for the full effect of $\mathrm{N}$ on NB lineage homeostasis, we used the NB-specific 1407-Gal4, type II NBspecific Pnt-Gal4, or IP-specific Erm-Gal4 drivers to overexpress $\mathrm{Su}(\mathrm{H})$ and mastermind (Mam), key genes in the canonical $\mathrm{N}$ pathway, and $\mathrm{Myc}$, a transcriptional target of $\mathrm{Su}(\mathrm{H})$ (Song and $\mathrm{Lu} 2011$ ). Compared with the controls, there was no significant change in the number of central brain NBs after these genetic manipulations (Fig. 1B,C; data not shown). Since overexpression of $\mathrm{Su}(\mathrm{H})$ or Mam was sufficient to activate canonical N signaling, as indicated by up-regulation of E(spl) expression (Supplemental Fig. S1A), these results suggest that activation of canonical $\mathrm{N}$ signaling under these conditions is in-

(c) 2013 Lee et al. This article is distributed exclusively by Cold Spring Harbor Laboratory Press for the first six months after the full-issue publication date (see http://genesdev.cshlp.org/site/misc/terms.xhtml). After six months, it is available under a Creative Commons License (AttributionNonCommercial 3.0 Unported), as described at http://creativecommons.org/ licenses/by-nc/3.0/. 
A

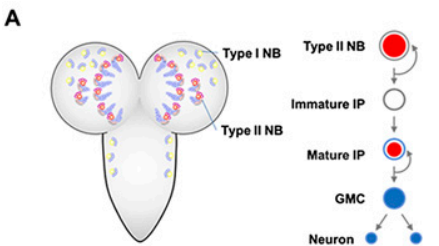

B

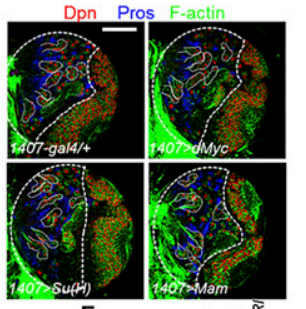

C

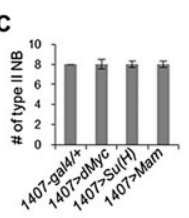

D Dpn pS505-dAKT

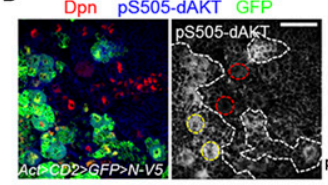

E

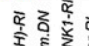

空色产

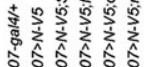

$\mathbf{F}$

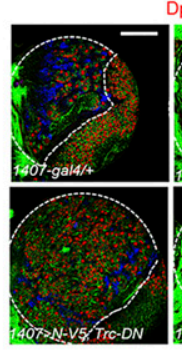
Dpn Pros F-actin
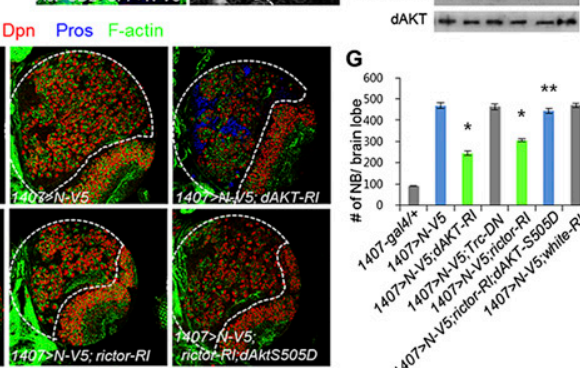

Figure 1. Notch regulates the mTORC2/AKT pathway in NBs. $(A)$ Diagram of Drosophila larval CNS showing type I and type II NBs in the central brain area (left) and the lineage hierarchy of type II NBs (right). (B) The effects of NB-specific overexpression of canonical Notch pathway components $\mathrm{Su}(\mathrm{H}), \mathrm{Mam}$, and dMyc. Larval brains at $120 \mathrm{~h}$ after larval hatching (ALH) were stained for Dpn (NBs), Pros (differentiated cells), and F-actin (cell cortex). Type II NB lineages are marked with fine white lines. In this and all subsequent figures, the central brain area is outlined with a bold white dashed line, and the $\mathrm{Dpn}^{+} \mathrm{NBs}$ within this area are quantified. (C) Quantification of data from $B$. (D) pS505-dAKT staining of GFP-marked NB flip-out clones overexpressing full-length $\mathrm{N}$ (N-v5). NBs within clones (circled in yellow) show an increased pS505-AKT signal compared with those outside of clones (circled in red). (E) Assay of mTORC2 activity by pS505-dAKT Western blot analysis of larva brain extracts after RNAi (RI) or dominant-negative (DN) transgene expression. Total dAKT was used as a loading control. $(F) \mathrm{N}$-induced NB expansion is blocked by inhibition of key components of the mTORC2/AKT pathway but not Trc-DN, and the effect of Rictor inhibition is restored by AKTS505D. (G) Quantification of data from E. $\left(^{\star}\right) P<0.0003$ (vs. $1407>N-V 5 /+) ;\left(^{\star \star}\right) P<0.002$ (vs. 1407 $>N-V 5$; rictor- $\left.R I\right)$ in Student's t-test; $n=10$. Bars: $A, E, 100 \mu \mathrm{m} ; C, 20 \mu \mathrm{m}$.

sufficient and that additional pathways are needed for the induction of ectopic NBs by $\mathrm{N}$ gain of function (GOF), as observed previously (Song and Lu 2011).

We next searched for other signaling events that may act together with the canonical $\mathrm{N}$ signaling pathway to mediate the effect of $\mathrm{N}$. We found that in the $\mathrm{N}$ GOF condition, there was a significant increase in the p$\mathrm{AKT}(\mathrm{S} 505)$ level, as measured by immunostaining and Western blot analyses (Fig. 1D,E). Since mTORC2 is the primary kinase responsible for AKT(S505) phosphorylation (Sarbassov et al. 2005; Hietakangas and Cohen 2007), this result indicated that mTORC2 is activated in the $\mathrm{N}$ GOF condition. Conversely, mTORC2 is inhibited in the $\mathrm{N}$ loss-of-function (LOF) condition (Supplemental Fig. S1B). No obvious change of p-AKT level was observed when Wingless or Hh signaling was altered (Supplemental Fig. S2), indicating specificity of the p-AKT response to $\mathrm{N}$ signaling. To assess the functional significance of mTORC2 activation, we inhibited Rictor (Hietakangas and Cohen 2007), a key component of mTORC2. Knockdown of rictor but not the control white $(W)$ gene significantly attenuated ectopic NB induction by N (Fig. 1F,G; Supplemental Fig. S3A). These data support that activation of mTORC2 contributes significantly to $\mathrm{N}$-induced NB overproliferation.

We next asked which mTORC2 effector is critically involved in NSC regulation by noncanonical N signaling. Intriguingly, in contrast to post-mitotic neurons in which Tricornered (Trc), but not AKT, acts as a key effector of mTORC2 to promote neuronal maintenance $(\mathrm{Wu}$ et al. 2013), NBs use AKT as the key mTORC2 effector for homeostasis control, since inhibiting AKT, but not Tre, prevented N GOF-induced ectopic NB formation (Fig. 1F,G), and a phospho-mimetic, constitutively active AKT (AKT-S505D) rescued the mTORC2 LOF effect on NSC homeostasis (Fig. 1F,G). These results support a critical role of the mTORC2/AKT axis in N-directed NSC homeostasis. Since canonical N signaling had no obvious effect on p-AKT(S505) level (Supplemental Fig. S1C,D), mTORC2 activation appears to be a specific effect of noncanonical $\mathrm{N}$ signaling.

We next sought to elucidate the signaling mechanism of the noncanonical $\mathrm{N}$ pathway that leads to mTORC2 activation. In flies and mammals, TOR kinase forms at least two complexes: mTORC1 and mTORC2. Compared with mTORC1, little is known about how mTORC2 is regulated by upstream signals (Zoncu et al. 2011) and its function in NSCs. Recent studies suggest that the functional state of mitochondria maintained by PINK1, a gene associated with Parkinson's disease (PD) and cancer (Devine et al. 2011), critically regulates mTORC2 activity and influences mitochondrial dynamics and function $(\mathrm{Wu}$ et al. 2013). Interestingly, dPINK1-null mutants exhibited defects in NB maintenance (Supplemental Fig. S4A,B). Furthermore, partial inhibition of PINK1 by RNAi could block the activation of mTORC2 and the formation of ectopic NBs induced by N GOF (Figs. 1E, 2A), although normal NBs were largely unaffected. RNAi-mediated inhibition of several other genes implicated in mitochondrial regulation, including ND-75 (respiratory chain complex-I [RCC-I] 75-kD subunit), PGC-1 $\alpha$ (biogenesis), and Drp1 (fission), also rescued the ectopic NB formation induced by N GOF without affecting normal NBs. By comparison, RNAi of $W$ or an RCC-III component failed to rescue the N GOF effect (Fig. 2A,B; Supplemental Fig. S3A). Genetic manipulations of PINK1 and the mitochondria-related genes also rescued the larval lethality induced by N GOF (Supplemental Fig. S4C). Treatment of N-GOF larvae with a small molecule inhibitor of Drp1 (Cassidy-Stone et al. 2008) induced mitochondrial fusion/aggregation (Supplemental Fig. S5A) and partially prevented ectopic NB formation and brain tumor formation (Fig. 2E,F). The role of mitochondrial fission was further evaluated by analyzing $d r p 1$-null mutant NB clones in which normal $\mathrm{NBs}$ were maintained, but activated $\mathrm{N}$-induced ectopic NBs were largely abolished (Fig. 2C,D).

To test the relevance of these findings to humans, we turned to glioblastoma multiforme (GBM), where altered $\mathrm{N}$ and mTOR signaling has been implicated (Dirks 2010; Cloughesy et al. 2013). Using patient-derived GBM lines that exhibited elevated $\mathrm{N}$ and PINK1 expression (Fig. 3K), $\mathrm{N}$ and mTORC2 signaling (Supplemental Fig. S6A), and RCC assembly (Supplemental Fig. S6B), we pharmacolog- 


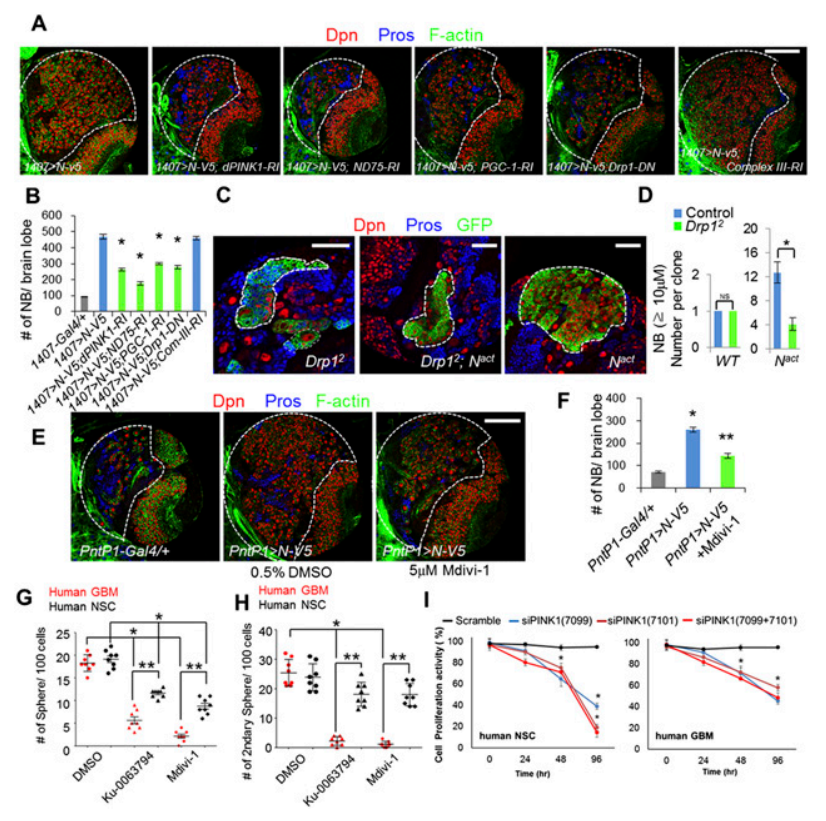

Figure 2. Involvement of PINK1 and other mitochondria-related genes in $\mathrm{N}$-induced NB overproliferation. (A) N-induced NB expansion is blocked by inhibiting genes involved in mitochondrial regulation. Larval brains at $120 \mathrm{~h}$ ALH were immunostained. $(B)$ Quantification of data from $A .\left(^{*}\right) P<0.0001$ versus $1407>N-V 5 /+; n=$ 10. Complex-III RNAi served as a specificity control. (C) Clonal analysis of NBs in the Drp1 mutant $\left(\operatorname{Drp} 1^{2}\right), \operatorname{Drp} 1^{2} ; N^{a c t}$, and $N^{a c t}$ backgrounds. MARCM clones are marked with GFP and outlined with white dashed lines. $(D)$ Quantification of data from $C$. (NS) Not significant; $\left({ }^{*}\right) P<0.001 ; n=5$. $(E) \mathrm{N}$-induced NB expansion is attenuated by Mdivi-1 treatment. $(F)$ Quantification of data from E. ${ }^{*} \mid P<0.001$ versus $\left.P n t P 1-G a 14 /+;{ }^{*}{ }^{*}\right) P<0.005$ versus $P n t P 1>N-V 5$; $n=5$. $(G, H)$ Primary $(G)$ and secondary $(H)$ neurosphere-forming activity of human GBM CSCs and normal fetal NSCs after chemical inhibition of Drp1 (Mdivi-1) or mTORC2 (Ku-0063794). $\left(^{*}\right) P<0.0001$ versus DMSO control; $\left(^{\star \star}\right) P<0.001$ human NSC versus human GBM. (I) Effects of lentiviral delivery of PINK1 shRNA (either singularly or with two shRNAs combined) on the proliferation of human NSCs and GBM cells. $\left(^{\star}\right) P<0.001$ versus scrambled shRNA control in Student's $t$-test. Bars: $A, E, 100 \mu \mathrm{m} ; C, 20 \mu \mathrm{m}$.

ically inhibited Drp1 (Cassidy-Stone et al. 2008), mTORC2 (Garcia-Martinez et al. 2009), or RCC-I. We found that growth and self-renewal of GBM CSCs were preferentially impaired by these treatments (Fig. 2G,H; Supplemental Fig. S5B,C), supporting a critical and conserved role of the newly identified noncanonical $\mathrm{N}$ signaling pathway in preserving CSCs. We also examined the effect of genetically inhibiting PINK1 in human NSC and GBM cell cultures. Consistent with PINK1 function being required in both normal NSCs and CSCs, shRNA-mediated knockdown of PINK1 inhibited cell proliferation in normal NSC and GBM CSC cultures (Fig. 2I).

We next tested the relationship between $\mathrm{N}$ and PINK1 in regulating the mTORC2/AKT axis. As reported before (Clark et al. 2006; Park et al. 2006; Yang et al. 2006), PINK1 LOF altered mitochondrial morphology and impaired indirect flight muscle integrity, as measured by the wing posture assay. These defects were effectively rescued by N GOF (Fig. 3A,B). Consistent with N playing an important role in regulating mitochondrial function, $\mathrm{N}$ LOF resulted in impaired RCC assembly and reduced ATP production (Fig. 3F; Supplemental Fig. S7A), and, as in the case of PINK1, N LOF in DA neurons caused mitochondrial aggregation and neuronal loss (Supplemental Fig. S7B,C), features associated with PD (Park et al. 2006; Yang et al. 2006). NB-specific knockdown of RCC-I $75 \mathrm{kD}$ also resulted in reduced NB number at the larval stage and loss of DA neurons in surviving adults (Supplemental Fig. S7D). These results suggested that mitochondrial function regulated by PINK1 and $\mathrm{N}$ is important for maintaining the NSCs during development and differentiated DA neurons in adults. Moreover, $N$ mutant mitochondria manifested morphological defects and reduced membrane potential in adult flight muscle (Fig. 3C-E). Importantly, N GOF efficiently rescued the PINK1 LOF effect on RCC assembly and ATP production, whereas PINK1 GOF failed to rescue the mitochondrial defects caused by N LOF (Fig. 3G; Supplemental S7A). In keeping with mitochondrial function being a key determinant of mTORC2 activation (Wu et al. 2013), PINK1 and $\mathrm{N}$ had similar effects on mTORC2 activity, as measured by p-AKT level, and N GOF restored mTORC2 activity in the PINK1 LOF condition (Fig. 3G). Intriguingly, PINK1 GOF also restored mTORC2 activity in the $\mathrm{N}$ LOF condition (Fig. 3G), and the coactivation of PINK1 and $\mathrm{N}$ had additive effects on mTORC2 activity (Supplemental Fig. S8A). Thus, although the functional assays support that $\mathrm{N}$ may act downstream from PINK1, the biochemical assays indicate that PINK1 and $\mathrm{N}$ have additive effects on mTORC2/AKT activation. PINK1 and $\mathrm{N}$ may have signaling branches that act independently to influence mTORC2 activity. Alternatively, PINK1 and N may act at the same level (e.g., by working in a protein complex rather than in a linear pathway) to regulate mTORC2 activation.

We further explored the mechanisms by which $\mathrm{N}$ and PINK1 interact to regulate mitochondrial function. By immunostaining with anti-N, the specificity of which was confirmed using the $N$ mutant (Supplemental Fig. $\mathrm{S} 8 \mathrm{~B}$ ), we found that endogenous $\mathrm{N}$ colocalized with the mito-GFP reporter (Supplemental Fig. S8B), and a N-GFP reporter expressed from the endogenous $N$ promoter colocalized with the mitochondrial marker Tom20 (Supplemental Fig. S8C). We also found that full-length $\mathrm{N}$ was present in Percoll gradient-purified mitochondria, whose purity was confirmed by the absence of various membrane markers (Fig. 3H; Supplemental Fig. S9A), and that $\mathrm{N}$ resided mostly at the outer membrane, as shown by fractionation studies (Supplemental Fig. S9B). These results support that $\mathrm{N}$ exerts its effect directly at the mitochondrial surface. Mammalian $\mathrm{N}$ was also found to localize to mitochondria (Supplemental Fig. S6D,E). Given that PINK1 can recruit proteins to the mitochondrial surface (Narendra et al. 2010), we next tested whether the mitochondrial localization of $\mathrm{N}$ is dependent on PINK1. The amount of mitochondria-bound $\mathrm{N}$, but not total $\mathrm{N}$, was reduced in the dPINK1 mutant but increased in the PINK1 GOF condition (Fig. 3I), consistent with PINK1 playing an active role in recruiting $\mathrm{N}$ to mitochondria. Intriguingly, although PINK1 can become stabilized on CCCP-damaged mitochondria and recruit proteins such as Parkin to these damaged organelles (Narendra et al. 2010), the amount of mitochondrial $\mathrm{N}$ was not affected by damage (Supplemental Fig. S9E). Importantly, in coimmunoprecipitation assays, PINK1 and $\mathrm{N}$ were found to physically associate in vivo (Fig. 3J; Supplemental Fig. S9C). Endogenous N-PINK1 in- 

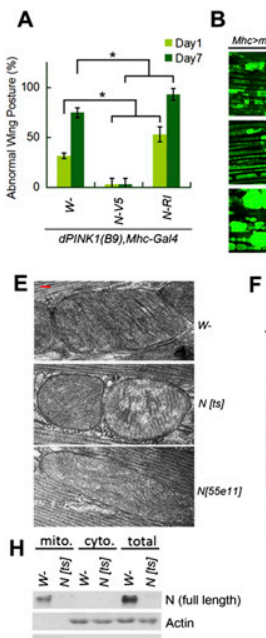

I
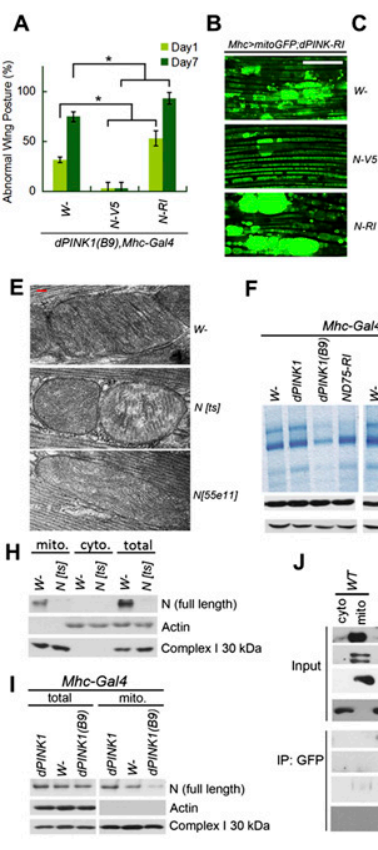

$\mathbf{F}$

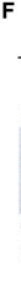

3
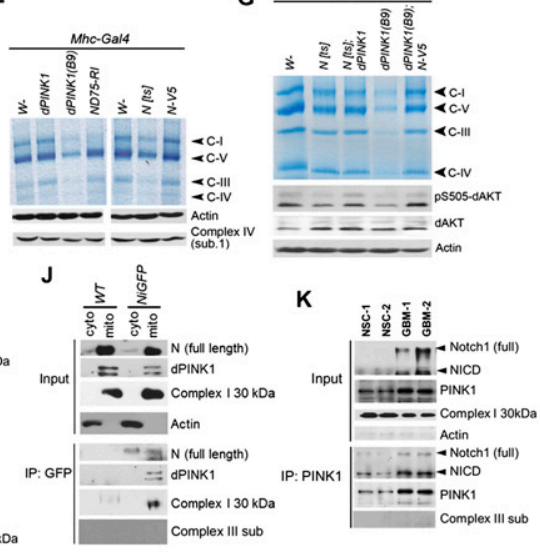

Figure 3. Genetic and biochemical interactions between $\mathrm{N}$ and PINK1. $(A, B)$ Effects of N overexpression or N RNAi on PINK1 LOFinduced abnormal wing posture $(A)$ and aggregation of muscle mitochondria $(B) .\left(^{\star}\right) P<0.0001$ versus $W^{-}$control in Student's $t$-test. (C) Effects of N LOF on muscle mitochondrial morphology monitored with mito-GFP. The uneven and enhanced mito-GFP signals suggest mitochondrial aggregation. $(D)$ Effects of $N$ mutations on mitochondrial membrane potential (indicated by JC-1 signal at 575-625 nm). The PINK1 mutant was used as a control. $(E)$ Transmission electron microscopy analysis of mitochondrial cristae morphology in $N$ mutants. Bar, $100 \mathrm{~nm}$. N[55e11] refers to N[55e11]/+ heterozygous females in $C-E$. $(F)$ Blue native gel (BNG) and Western blot analyses of RCC assembly in PINK1 and N LOF and GOF backgrounds. Actin and the complex IV subunit 1 served as loading controls. $(G)$ Effects of N GOF on RCC assembly and pS505dAKT level changes caused by PINK1 LOF. $(H, I)$ Fractionation assay showing enrichment of full-length $\mathrm{N}$ at mitochondria $(H)$ and increased mitochondrial $\mathrm{N}$ in the PINK1 overexpression condition $(I)$. (J) Coimmunoprecipitation assays using NiGFP fly head tissue to demonstrate $\mathrm{N}$ interaction with PINK1 and complex I $30 \mathrm{kD}$. The complex III subunit served as a negative control. $(K)$ The interaction between hNotch1 and PINK1 in the mitochondria of human NSCs and GBM cells. The complex III subunit served as a negative control. GBM cells show elevated expression of PINK1 and both the fulllength and the intracellular domain of Notch1.

teraction was also observed in human GBM cells (Fig. 3K). Moreover, we detected $\mathrm{N}$ association with a RCC-I 30-kD subunit, which was previously shown to interact with PINK1 (Wu et al. 2013), but no $\mathrm{N}$ association with the RCC-III or RCC-IV subunits (Fig. 3J). These results reveal a conserved noncanonical mechanism whereby $\mathrm{N}$ regulates mitochondrial function and mTORC2 activity through direct interaction with PINK1 and specific RCC subunits under physiological conditions.

We next tested the relationship between the newly identified noncanonical $\mathrm{N}$ signaling pathway and the canonical $\mathrm{N}$ pathway in $\mathrm{N}$-directed NB homeostasis. Although knocking down the canonical or noncanonical $\mathrm{N}$ signaling pathway individually only partially rescued the N GOF-induced brain tumor phenotype, combined knockdown of both pathways resulted in nearly complete rescue (Fig. 4A,B). Strikingly, while RNAi-mediated in- hibition of either pathway alone had little effect on normal NB maintenance, their combined knockdown significantly reduced the number of normal NBs (Fig. 4C,D), while their combination with control W RNAi had no effect (Fig. 4C; Supplemental Fig. S3C), supporting the importance of both pathways in maintaining normal NBs. Moreover, although the GOF of genes in the noncanonical pathway alone had no obvious effect on NB number, they strongly enhanced NB expansion induced by $\mathrm{N}$ GOF (Fig. 4E,F). Furthermore, combining the inhibition of mitochondrial fusion regulator Marf (Deng et al. 2008), which impinges on mitochondria and activates the noncanonical pathway (Supplemental Fig. S9D), with the GOF of dMyc, a key target of the canonical pathway (Song and Lu 2011), resulted in full rescue of $\mathrm{N}$ LOF-induced type II NB loss (Fig. 4G,H). By comparison, the coexpression of GFP or Parkin, another gene involved in mitochondrial regulation, failed to modify the rescuing effect of dMyc (Fig. 4H; Supplemental Fig. S9F). Thus, canonical and noncanonical $\mathrm{N}$ signaling pathways both play important and specific roles in mediating the effects of N on NSC homeostasis.

Finally, we tested whether coactivation of the canonical and noncanonical $\mathrm{N}$ signaling pathways is sufficient to induce ectopic NBs, hence recapitulating the effect of N GOF. Activation of the canonical pathway via $\mathrm{Su}(\mathrm{H})$ GOF promoted nucleolar growth of IPs (Supplemental Fig. S1D), which is likely mediated by dMyc, a known master regulator of nucleolar growth (Song and Lu 2011). However, no ectopic NB was formed when $\mathrm{Su}(\mathrm{H})$ was overexpressed using the IP-specific Erm-Gal4 driver (Fig. $4 \mathrm{I}, \mathrm{J})$. In contrast, coexpression of $\mathrm{Su}(\mathrm{H})$ and AKT-S505D under the control of Erm-Gal4 promoted the dedifferentiation of IPs into ectopic NBs resembling those found in the N GOF condition (Fig. 4I,J), although AKT-S505D overexpression alone did not affect nucleolar growth (Supplemental Fig. S1E) and had no obvious effect on type II NB number (Fig. 4I,J). Similar results were obtained when $\mathrm{Su}(\mathrm{H})$ or Mam were coexpressed with PINK1 (Supplemental Fig. S10A,C). We also used a N $\Delta$ cdc10 construct defective in $\mathrm{Su}(\mathrm{H})$ binding and thus canonical $\mathrm{N}$ signaling (Lawrence et al. 2000) but capable of noncanonical N signaling (Supplemental Fig. S1A). Coexpression of $\mathrm{Su}(\mathrm{H})$ and $\mathrm{N} \Delta \mathrm{cdc} 10$ also induced ectopic NBs (Supplemental Fig. S10B,D). These results suggest that noncanonical and canonical $\mathrm{N}$ signaling mediate distinct aspects of $\mathrm{N}$ function and act coordinately to regulate NSC behavior.

Our results uncover a novel mechanism of $\mathrm{N}$ in regulating NSC self-renewal and maintenance through a noncanonical signaling pathway involving PINK1, mTORC2, and AKT. A central feature of this noncanonical N signaling pathway is specific mitochondrial roles of $\mathrm{N}$ in regulating RCC function through direct interactions with PINK1 and select RCC subunits and in activating mTORC2. $\mathrm{N}$ could act through a number of possible mechanisms, such as facilitating the import or assembly of RCC components (Ades and Butow 1980), as suggested by its interaction with complex I subunits (Fig. 3J), or directing the quality control of mitochondria, as has been implicated for PINK1 (Rugarli and Langer 2012). Future studies will determine the exact domains of $\mathrm{N}$ involved in PINK1 interaction and whether noncanonical $\mathrm{N}$ signaling is ligand-dependent. Although the exact mechanism remains to be determined, our results will help us to 


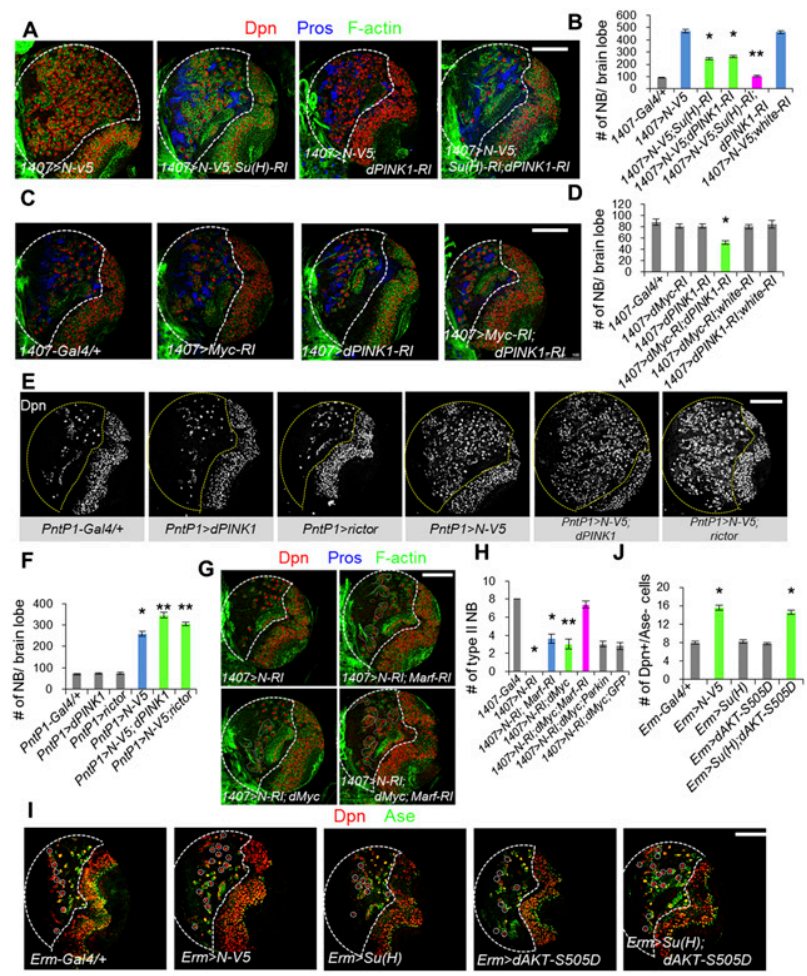

Figure 4. The interaction between canonical and noncanonical $\mathrm{N}$ signaling on NB homeostasis. $(A)$ The effects of combined RNAi of $\mathrm{Su}(\mathrm{H})$ and PINK1 on N GOF-induced NB expansion. $(B)$ Quantification of data from $A .\left(^{\star}\right) P<0.0002$ versus $\left.1407>N-V 5 /+\left._{;}\right|^{\star \star}\right) P<0.0005$ versus $1407>N-V 5$; Su(H)-RI or $1407>N-V 5 ; d P I N K 1-R I ; n=5 .(C)$ The effects of combined RNAi of Myc and PINK1 on normal NB number. $(D)$ Quantification of data from $C .\left(^{*}\right) P<0.001$ versus $1407>d M y c-R I$ or $1407>d P I N K 1-R I ; n=5$. $(E)$ The effects of coexpression of PINK1 and Rictor on N GOF-induced NB expansion. $(F)$ Quantification of data from $E .\left(^{*}\right) P<0.0001$ versus PntP1-Gal4/+ control; $^{* \star}{ }^{\star *} P<0.05$ versus $P n t P 1>N-V 5 /+; n=5$. $(G)$ Synergy between dMyc overexpression and Marf-RI in rescuing N LOFinduced type II NB loss. (H) Quantification of data from $G$. ( $\left.{ }^{*}\right) P<$ 0.005 versus $\left.1407>N-R I /+;\left.\right|^{\star *}\right) P<0.05$ versus $1407>N-R I ; d M y c$ or $1407>N-R I ; M a r f-R I ; n=5$. Parkin or GFP coexpression served as a specificity control. (I) The effects of coexpression of $\mathrm{Su}(\mathrm{H})$ and dAKT-S505D in mature IPs (driven by Erm-gal4). The ectopic NBs dedifferentiated from mature IPs are identified by marker expression $\left(\mathrm{Dpn}^{+}\right.$and $\left.\mathrm{Ase}^{-}\right)$and marked by white dashed circles. (J) Quantification of data from $I .\left(^{\star}\right) P<0.0002$ versus Erm-Gal4/+ control in Student's $t$-test; $n=5$. Bars, $100 \mu \mathrm{m}$.

understand earlier observations in Drosophila that mutations in $N$ affected mitochondrial respiration (Thorig et al. 1981) and data from mammalian systems implicating $\mathrm{N}$ in mitochondrial and metabolic regulation (Dotti et al. 2004; Landor et al. 2011). The physiological significance of this newly defined noncanonical $\mathrm{N}$ pathway is also underscored by the phenotypes of various diseases associated with $\mathrm{N}$ dysregulation. For example, cerebral autosomal dominant arteriopathy with subcortical infarcts and leukoencephalopathy (CADASIL), a disease caused by mutations in NOTCH3 (Joutel et al. 1996), is associated with mitochondrial impairment (Dotti et al. 2004). On the other hand, GOF mutations of Notch1 are implicated in over half of human T-cell acute lymphoblastic leukemia (T-ALL) (Weng et al. 2004), in which a pathogenic role of mTORC2 has been proposed, although how $\mathrm{N}$ impinges on mTORC2 in this setting is unknown (Lee et al. 2012). Mammalian $\mathrm{N}$ has been shown to act through AKT and mitochondria to promote T-cell survival (Perumalsamy et al. 2010), although the mechanism is distinct from the one uncovered here. Finally, mTORC2 was shown to be required for the selfrenewal and maintenance of CSCs but dispensable in normal stem cells (Zoncu et al. 2011). Our findings that CSC-like brain tumor-forming cells are particularly dependent on the noncanonical $\mathrm{N}$ pathway in flies and humans identify the newly discovered noncanonical $\mathrm{N}$ signaling pathway as a potential target for disease intervention.

\section{Materials and methods}

\section{Fly genetics}

Fly culture and crosses were performed according to standard procedures and were raised at the indicated temperatures. Drosophila stocks used in this study were $d P I N K 1^{B 9}$ (J.K. Chung), Drp $1^{2}$ (H. Bellen), $N^{55 e 11} ; N i G F P /+$ (F. Schweisguth), 1407-Gal4 (L. Luo), PntP1-Gal4 (Y.N. Jan), Erm-Gal4 (C.Y. Lee and G. Rubin), Mhc-Gal4 (T. Littleton), and UAS-N-V5 (M. Fortini). dPINK1, dPINK1-RNAi, and Parkin transgenes were described previously (Yang et al. 2006). The other transgenes were $d M y c$ (F. Demontis and B. Edgar), rictor, dAKT-S505D (S. Cohen), mitoGFP (W. Saxton), Marf-RNAi (M. Guo), white-RNAi (D. Smith), NECN (deletion of the entire intracellular domain of N), NAcdc10 [deletion of the intracellular domain of $\mathrm{N}$ comprising the $\mathrm{Su}(\mathrm{H})$ interacting region] (E. Giniger), ND75-RNAi (2286-R-3) (National Institute of Genetics Fly Stock Center, Japan), Complex III-RNAi (v33015), dMyc-RNAi (v106066), dMyc-RNAi-2 (v17487), N-RNAi (v1112 and v27229), and Dicer2 (v60008; Vienna Drosophila RNAi Center). Mam-WT (B27743), Su(H)myc (B5814), $M a m-D N$ (dominant negative) (B26672), Trc-DN (B32086), Su(H)-RNAi (B28900), rictor-RNAi (B31388), rictor-RNAi (B31527), dAKT-RNAi (B33615), dPGC1 $\alpha$-RNAi (B33914), Notch ${ }^{\text {ts }}$ (B2533), Shaggy-DN (B5255), TCF- $\Delta N$ (B4784), Smoothened-RNAi (B27037), and all other stocks were obtained from Bloomington Drosophila Stock Center. To enhance the efficiency of $\mathrm{N}$ knockdown, Dicer2 was coexpressed with $N$-RNAi (Song and $\mathrm{Lu} 2011$ ).

\section{MARCM and flip-out clonal analysis}

To generate NB MARCM clones and overexpression clones, $24 \mathrm{~h}$ after larval hatching (ALH), larvae were heat-shocked for $90 \mathrm{~min}$ at $37^{\circ} \mathrm{C}$ and further aged for $72 \mathrm{~h}$ at $25^{\circ} \mathrm{C}$ before dissection. MARCM analyses were performed essentially as described (Song and Lu 2011). For making overexpression clones, w, hsFLP; Actin 5c>CD2>Gal4, UAS-GFP-NLS was crossed with the indicated UAS lines, and, $24 \mathrm{~h} \mathrm{ALH}$, larvae were heat-shocked for $90 \mathrm{~min}$ at $37^{\circ} \mathrm{C}$ and further aged for $72 \mathrm{~h}$ at $25^{\circ} \mathrm{C}$ before dissection.

\section{Abnormal wing posture}

Abnormal wing posture was analyzed as described (Yang et al. 2006). Briefly, the number of flies with abnormal wing posture (either held up or drooped) was scored after male flies of the indicated genotypes were aged for $14 \mathrm{~d}$ at $29^{\circ} \mathrm{C}$. For each experiment, at least 60 flies in three separate vials were scored, and the percentage of flies with abnormal wing posture was presented for each genotype.

\section{ATP measurement}

The ATP level in Drosophila thoracic muscle was measured essentially as previously described (Wu et al. 2013) using a luciferase-based bioluminescence assay (ATP Bioluminescence assay kit HS II, Roche Applied Science).

\section{Statistical analysis}

The statistical significance of all data was evaluated by unpaired Student's t-tests. 


\section{Acknowledgments}

We are grateful to Dr. J.K. Chung, Dr. H. Bellen, Dr. E. Giniger, Dr. F. Schweisguth, Dr. L. Luo, Dr. Y.N. Jan, Dr. C.Y. Lee, Dr. G. Rubin, Dr. T. Littleton, Dr. M. Fortini, Dr. F. Demontis, Dr. B. Edgar, Dr. S. Cohen, Dr. W. Saxton, Dr. M. Guo, Dr. J. Skeath, Dr. L. Pallanck, University of Iowa Developmental Studies Hybridoma Bank, Bloomington Drosophila Stock Center, National Institute of Genetics Fly Stock Center Japan, and Vienna Drosophila RNAi Center for fly stocks and reagents. We thank J. Gaunce and G. Silverio for technical assistance, and members of the Lu laboratory for discussions and help. This work was supported by National Institutions of Health grants (R01 NS043167 and R01 AR054926) to B.L., and a Stanford University School of Medicine Dean's Post-doctoral Fellowship to Z.W.

\section{References}

Ades IZ, Butow RA. 1980. The products of mitochondria-bound cytoplasmic polysomes in yeast. I Biol Chem 255: 9918-9924.

Andersson ER, Sandberg R, Lendahl U. 2011. Notch signaling: Simplicity in design, versatility in function. Development 138: 35933612.

Artavanis-Tsakonas S, Muskavitch MA. 2010. Notch: The past, the present, and the future. Curr Top Dev Biol 92: 1-29.

Bowman SK, Rolland V, Betschinger J, Kinsey KA, Emery G, Knoblich JA. 2008. The tumor suppressors Brat and Numb regulate transitamplifying neuroblast lineages in Drosophila. Dev Cell 14: 535546.

Cassidy-Stone A, Chipuk JE, Ingerman E, Song C, Yoo C, Kuwana T, Kurth MJ, Shaw JT, Hinshaw JE, Green DR, et al. 2008. Chemical inhibition of the mitochondrial division dynamin reveals its role in Bax/Bak-dependent mitochondrial outer membrane permeabilization. Dev Cell 14: 193-204.

Clark IE, Dodson MW, Jiang C, Cao JH, Huh JR, Seol JH, Yoo SJ, Hay BA, Guo M. 2006. Drosophila pink1 is required for mitochondrial function and interacts genetically with parkin. Nature 441: 11621166.

Cloughesy TF, Cavenee WK, Mischel PS. 2013. Glioblastoma: From molecular pathology to targeted treatment. Annu Rev Pathol doi: 10.1146/annurev-pathol-01111-130324.

Deng H, Dodson MW, Huang H, Guo M. 2008. The Parkinson's disease genes pink1 and parkin promote mitochondrial fission and/or inhibit fusion in Drosophila. Proc Natl Acad Sci 105: 14503-14508.

Devine MJ, Plun-Favreau H, Wood NW. 2011. Parkinson's disease and cancer: Two wars, one front. Nat Rev Cancer 11: 812-823.

Dirks PB. 2010. Brain tumor stem cells: The cancer stem cell hypothesis writ large. Mol Oncol 4: 420-430.

Doe CQ. 2008. Neural stem cells: Balancing self-renewal with differentiation. Development 135: 1575-1587.

Dotti MT, De Stefano N, Bianchi S, Malandrini A, Battisti C, Cardaioli E, Federico A. 2004. A novel NOTCH3 frameshift deletion and mitochondrial abnormalities in a patient with CADASIL. Arch Neurol 61: 942-945.

Garcia-Martinez JM, Moran J, Clarke RG, Gray A, Cosulich SC, Chresta CM, Alessi DR. 2009. Ku-0063794 is a specific inhibitor of the mammalian target of rapamycin (mTOR). Biochem J 421: 29-42.

Hietakangas V, Cohen SM. 2007. Re-evaluating AKT regulation: Role of TOR complex 2 in tissue growth. Genes Dev 21: 632-637.

Joutel A, Corpechot C, Ducros A, Vahedi K, Chabriat H, Mouton P, Alamowitch S, Domenga V, Cecillion M, Marechal E, et al. 1996. Notch3 mutations in CADASIL, a hereditary adult-onset condition causing stroke and dementia. Nature 383: 707-710.

Knoblich JA. 2010. Asymmetric cell division: Recent developments and their implications for tumour biology. Nat Rev Mol Cell Biol 11: 849860.

Landor SK, Mutvei AP, Mamaeva V, Jin S, Busk M, Borra R, Gronroos TJ, Kronqvist P, Lendahl U, Sahlgren CM. 2011. Hypo- and hyperactivated Notch signaling induce a glycolytic switch through distinct mechanisms. Proc Natl Acad Sci 108: 18814-18819.

Lawrence N, Klein T, Brennan K, Martinez Arias A. 2000. Structural requirements for Notch signalling with Delta and serrate during the development and patterning of the wing disc of Drosophila. Development 127: 3185-3195.
Lee K, Nam KT, Cho SH, Gudapati P, Hwang Y, Park DS, Potter R, Chen J, Volanakis E, Boothby M. 2012. Vital roles of mTOR complex 2 in Notch-driven thymocyte differentiation and leukemia. I Exp Med 209: 713-728.

Liu C, Zong H. 2012. Developmental origins of brain tumors. Curr Opin Neurobiol 22: 844-849.

Morrison SJ, Kimble J. 2006. Asymmetric and symmetric stem-cell divisions in development and cancer. Nature 441: 1068-1074.

Narendra DP, Jin SM, Tanaka A, Suen DF, Gautier CA, Shen J, Cookson MR, Youle RJ. 2010. PINK1 is selectively stabilized on impaired mitochondria to activate parkin. PLOS Biol 8: e1000298.

Park J, Lee SB, Lee S, Kim Y, Song S, Kim S, Bae E, Kim J, Shong M, Kim JM, et al. 2006. Mitochondrial dysfunction in Drosophila PINK1 mutants is complemented by parkin. Nature 441: 1157-1161.

Perumalsamy LR, Nagala M, Sarin A. 2010. Notch-activated signaling cascade interacts with mitochondrial remodeling proteins to regulate cell survival. Proc Natl Acad Sci 107: 6882-6887.

Rugarli EI, Langer T. 2012. Mitochondrial quality control: A matter of life and death for neurons. EMBO I 31: 1336-1349.

Sarbassov DD, Guertin DA, Ali SM, Sabatini DM. 2005. Phosphorylation and regulation of Akt/PKB by the rictor-mTOR complex. Science 307: 1098-1101.

Song Y, Lu B. 2011. Regulation of cell growth by Notch signaling and its differential requirement in normal vs. tumor-forming stem cells in Drosophila. Genes Dev 25: 2644-2658.

Sousa-Nunes R, Cheng LY, Gould AP. 2010. Regulating neural proliferation in the Drosophila CNS. Curr Opin Neurobiol 20: 50-57.

Thorig GE, Heinstra PW, Scharloo W. 1981. The action of the notchlocus in Drosophila melanogaster. II. Biochemical effects of recessive lethals on mitochondrial enzymes. Genetics 99: 65-74.

Wang H, Somers GW, Bashirullah A, Heberlein U, Yu F, Chia W. 2006. Aurora-A acts as a tumor suppressor and regulates self-renewal of Drosophila neuroblasts. Genes Dev 20: 3453-3463.

Weng AP, Ferrando AA, Lee W, Morris JPt, Silverman LB, Sanchez-Irizarry C, Blacklow SC, Look AT, Aster JC. 2004. Activating mutations of NOTCH1 in human T cell acute lymphoblastic leukemia. Science 306: 269-271.

Weng M, Golden KL, Lee CY. 2010. dFezf/Earmuff maintains the restricted developmental potential of intermediate neural progenitors in Drosophila. Dev Cell 18: 126-135.

Wu Z, Sawada T, Shiba K, Liu S, Kanao T, Takahashi R, Hattori N, Imai Y, Lu B. 2013. Tricornered/NDR kinase signaling mediates PINK1directed mitochondrial quality control and tissue maintenance. Genes Dev 27: 157-162.

Yang Y, Gehrke S, Imai Y, Huang Z, Ouyang Y, Wang JW, Yang L, Beal MF, Vogel H, Lu B. 2006. Mitochondrial pathology and muscle and dopaminergic neuron degeneration caused by inactivation of Drosophila Pink1 is rescued by Parkin. Proc Natl Acad Sci 103: 1079310798.

Zhong W, Chia W. 2008. Neurogenesis and asymmetric cell division. Curr Opin Neurobiol 18: 4-11.

Zoncu R, Efeyan A, Sabatini DM. 2011. mTOR: From growth signal integration to cancer, diabetes and ageing. Nat Rev Mol Cell Biol 12: $21-35$. 


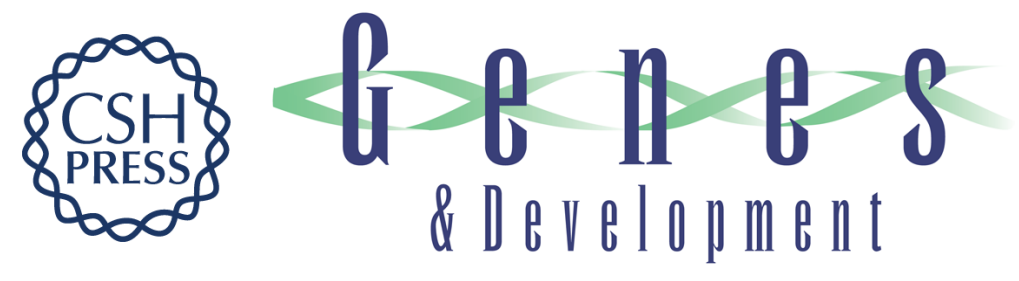

\section{Roles of PINK1, mTORC2, and mitochondria in preserving brain tumor-forming stem cells in a noncanonical Notch signaling pathway}

Kyu-Sun Lee, Zhihao Wu, Yan Song, et al.

Genes Dev. 2013, 27:

Access the most recent version at doi:10.1101/gad.225169.113

Supplemental http://genesdev.cshlp.org/content/suppl/2013/12/18/27.24.2642.DC1
Material

References This article cites 35 articles, 18 of which can be accessed free at:

http://genesdev.cshlp.org/content/27/24/2642.full.html\#ref-list-1

Creative This article is distributed exclusively by Cold Spring Harbor Laboratory Press for the first

Commons six months after the full-issue publication date (see

License http://genesdev.cshlp.org/site/misc/terms.xhtml). After six months, it is available under a Creative Commons License (Attribution-NonCommercial 3.0 Unported), as described at http://creativecommons.org/licenses/by-nc/3.0/.

Email Alerting Receive free email alerts when new articles cite this article - sign up in the box at the top Service right corner of the article or click here.

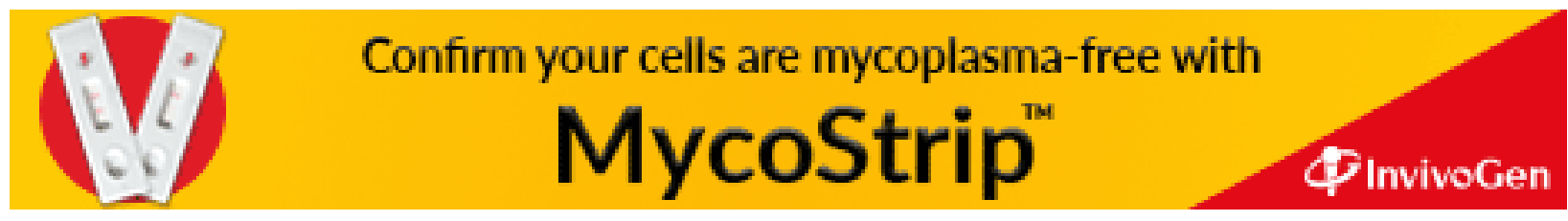

